Ferroelectrics 342:73-82, 2006

\title{
Computational Modeling of Ferromagnetic Shape Memory Thin Films
}

\author{
J. Liakhova ${ }^{2}$, M. Luskin ${ }^{1}$, and T. Zhang ${ }^{2}$ \\ ${ }^{1}$ School of Mathematics \\ 206 Church St. SE \\ University of Minnesota \\ Minneapolis, MN 55455, USA \\ Email: luskin@umn.edu \\ ${ }^{2}$ School of Mathematics \\ 206 Church St. SE \\ University of Minnesota \\ Minneapolis, MN 55455, USA
}

We propose a mathematical model for the deflection of single crystal films of $\mathrm{Ni}_{2} \mathrm{MnGa}$ ferromagnetic shape memory alloys in response to the application of a magnetic field. We then present the results of numerical simulations obtained from the finite element approximation of this model to study the deflection of the film due to the application of a magnetic field.

Keywords: ferromagnetic, shape memory, active thin film, computational modeling

\section{INTRODUCTION}

The $\mathrm{Ni}_{2} \mathrm{MnGa}$ ferromagnetic shape memory (FSM) alloy undergoes a cubic to tetragonal structural phase transformation that exhibits the shape memory effect, and it is also ferromagnetic at temperatures below its structural martensitic transformation temperature [1,2]. Because the three tetragonal variants of martensite have orthogonal easy axes of magnetization, and the typical rotations of variants are small in a compatible microstructure, a reversible shape change can be produced by applying appropriate magnetic fields to rearrange the variants [1].

Theory indicates that single crystal films will have significant advantages over polycrystalline films for producing motion [1], and single crystal films of $\mathrm{Ni}_{2} \mathrm{MnGa}$ that exhibit the ferromagnetic shape memory effect have recently been grown [2]. It has also been demonstrated that the transformation temperature of single crystal films of $\mathrm{Ni}_{2} \mathrm{MnGa}$ can be raised above room temperature by slightly varying the composition of $\mathrm{Ni}_{2} \mathrm{MnGa}$ from its stoichiometric composition [2].

We propose a model for the deformation of single crystal ferromagnetic shape memory films in response to the application of a magnetic field. We use this general model to develop a model for single crystal $\mathrm{Ni}_{2} \mathrm{MnGa}$ films at temperatures below its structural transformation temperature, and we then present the results of numerical simulations obtained from the finite element approximation of this model to study the deflection of the film by the application of a magnetic field. 


\section{BULK MODEL FOR FERROMAGNETIC SHAPE MEMORY CRYSTALS}

We denote the reference crystal domain for a ferromagnetic shape memory crystal with thickness $\delta<<1$ by $\Omega_{\delta}=\left\{\left(x_{1}, x_{2}, x_{3}\right):\left(x_{1}, x_{2}\right) \in S,-\delta / 2 \leq x_{3} \leq \delta / 2\right\}$ where $S \subseteq \mathrm{R}^{2}$ is a two dimensional region (see Figure 1). Deformations of the crystal will be denoted by $u(x): \Omega_{\delta} \rightarrow \mathrm{R}^{3}$, the deformed crystal by $u\left(\Omega_{\delta}\right)$, the magnetization of the crystal by $m(z): u\left(\Omega_{\delta}\right) \rightarrow \mathrm{R}^{3}$, the scalar potential of the associated magnetic field by $\zeta(z): \mathrm{R}^{3} \rightarrow \mathrm{R}$, the applied magnetic field by $h(z): \mathrm{R}^{3} \rightarrow \mathrm{R}^{3}$, and the temperature by $\theta$. We note that the deformation $u(x)$ is naturally defined in material coordinates; while the magnetization $m(z)$, scalar potential $\zeta(z)$, and applied magnetic field $h(z)$ are naturally defined in spatial coordinates.
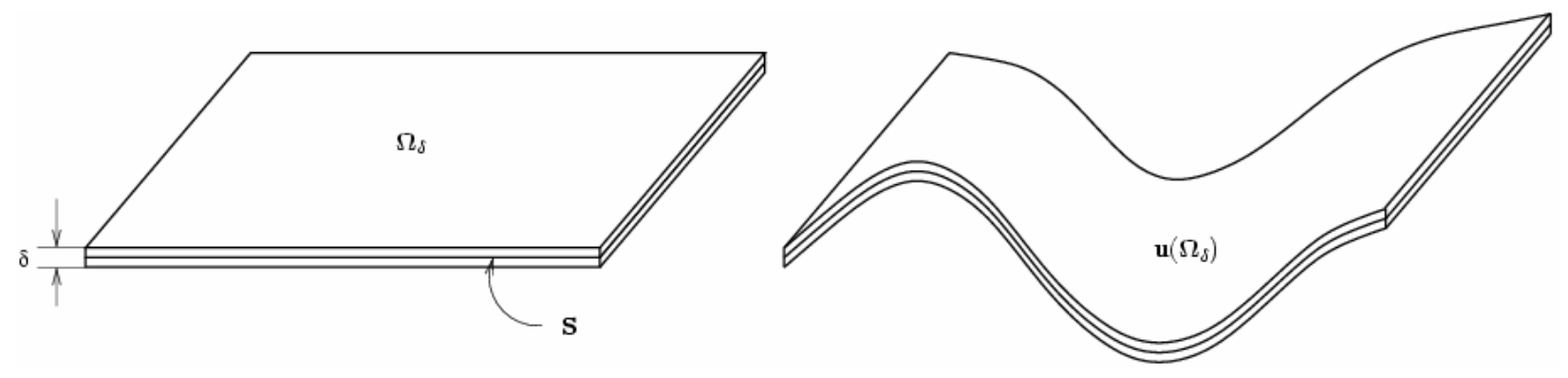

Figure 1. Reference crystal domain $\Omega_{\delta}$ and deformed crystal domain $u\left(\Omega_{\delta}\right)$.

The free energy of a ferromagnetic shape memory crystal with deformation $u(x): \Omega_{\delta} \rightarrow \mathrm{R}^{3}$ and magnetization $m(z): u\left(\Omega_{\delta}\right) \rightarrow \mathrm{R}^{3}$ can be modeled by [3]

$$
\begin{aligned}
& e^{(\delta)}(u, m)=\int_{\Omega_{\delta}}\left\{\kappa\left|D^{2} u(x)\right|^{2}+\varphi(\nabla u(x), m(u(x)), \theta)\right\} d x \\
& \quad+\int_{u\left(\Omega_{\delta}\right)}\left\{\mu\left|\nabla_{z} m(z)\right|^{2}-h(z) \cdot m(z)\right\} d z+e_{\text {mag }}^{(\delta)}(u, m) \\
& =\int_{\Omega_{\delta}}\left\{\kappa\left|D^{2} u(x)\right|^{2}+\varphi(\nabla u(x), m(u(x)), \theta)\right\} d x \\
& \quad+\int_{\Omega_{\delta}}\left\{\mu\left|\nabla_{z} m(u(x))\right|^{2}-h(u(x)) \cdot m(u(x))\right\} \operatorname{det} \nabla u(x) d x+e_{\text {mag }}^{(\delta)}(u, m)
\end{aligned}
$$

where $\kappa$ is the surface energy coefficient, $\varphi$ is the sum of the elastic and anisotropic free energy densities, $\mu$ is the exchange energy coefficient, and $e_{m a g}^{(\delta)}(u, m)$ is the magnetostatic energy given by

with

$$
e_{m a g}^{(\delta)}(u, m)=\frac{1}{2} \int_{\mathrm{R}^{3}}|\nabla \zeta(z)|^{2} d z
$$

$$
\nabla \cdot(-\nabla \zeta+m)=0 \quad \text { for } \mathrm{z} \in \mathrm{R}^{3}
$$


where the magnetization $m$ has been extended by zero outside of the crystal domain $u\left(\Omega_{\delta}\right)$. The magnetization satisfies the magnetic saturation condition

$$
|m(u(x))| \operatorname{det} \nabla u(x)=m_{s}(\theta) \text { for } x \in \Omega_{\delta},
$$

where the saturation magnetization $m_{s}(\theta)>0$ for temperatures $\theta$ below the structural transformation temperature. However, we will use the simpler condition

$$
|m(u(x))|=m_{s}(\theta) \quad \text { for } \quad x \in \Omega_{\delta}
$$

without significant loss of accuracy.

The terms in the free energy (2.1) represent, from left to right, the surface energy, the combined elastic and magnetic anisotropy energy, the exchange energy, the interaction energy due to the applied magnetic field, and the magnetostatic energy.

We will assume that the film is released from the substrate and free on its top and bottom lateral surfaces, $S \times\{-\delta / 2, \delta / 2\}$, but that it is attached to the substrate on the edges of the film $\partial S \times(-\delta / 2, \delta / 2)$ where $\partial S$ denotes the boundary of the reference domain region $S$. More general boundary conditions can also be treated by our model.

The surface energy term above is defined by

$$
\kappa \int_{\Omega_{\delta}}\left|D^{2} u(x)\right|^{2} d x=\kappa \int_{\Omega_{\delta}}\left[\sum_{i, j=1}^{3}\left|\frac{\partial^{2} u(x)}{\partial x_{i} \partial x_{j}}\right|^{2}\right] d x .
$$

This "strain gradient" surface energy gives an infinite energy if the deformation gradient is discontinuous across an interface. We have introduced an alternative "total variation" surface energy in $[3,4,5]$ that gives a finite surface energy that is proportional to the surface area of interfaces across which the deformation gradient is discontinuous. The total variation of a deformation gradient $\nabla u$ that is discontinuous across the piecewise smooth surfaces $\sigma_{j}$ for $j=1, \ldots, J$, separating the open sets $\omega_{\ell}$ in the disjoint union $\Omega_{\delta}=\sum_{\ell=1}^{L} \omega_{\ell}$ is given by

$$
\int_{\Omega_{\delta}}|D(\nabla u)|=\sum_{j=1}^{J} \int_{\sigma_{j}}\left|\llbracket \nabla u \rrbracket_{\sigma_{j}}\right| d S+\sum_{\ell=1}^{L} \int_{\omega_{\ell}} \sqrt{\sum_{m, n=1}^{3}\left(\frac{\partial^{2} u}{\partial x_{m} \partial x_{n}}\right)^{2}} d x
$$

where $\llbracket \nabla u \rrbracket_{\sigma_{j}}$ denotes the jump of the deformation gradient across the interface $\sigma_{j}$.

We will show later in this paper that the formula (2.3) gives the simple expression (4.2) when applied to continuous, piecewise linear finite element functions.

Not only is the "total variation" surface energy a more accurate model for the energy of martensitic crystals with microstructure, but it allows the use of continuous, piecewise linear finite element approximation to the deformation rather than the more complex finite element methods needed to approximate a "strain gradient" surface energy [4,5]. We used this more accurate "total variation" sharp interface surface energy in the computations presented in this paper, but we will use the "strain gradient" surface energy in some of the discussion of this paper for simplicity of exposition.

We will model the elastic and anisotropic free energy density for a ferromagnetic shape memory crystal by a continuous and frame-indifferent function $\varphi$ with the symmetry of the high temperature austenitic phase $[7,8]$. The frame indifference property $[7,9]$ is that

$$
\varphi(R F, R m, \theta)=\varphi(F, m, \theta), \quad \text { for all } R \in S O(3) \text {, }
$$


where $S O(3)$ is the group of proper rotations, and the crystal symmetry property $[7,8,9]$ is that

$$
\varphi(F Q, m, \theta)=\varphi(F, m, \theta), \quad \text { for all } Q \in \mathrm{G},
$$

where $\mathrm{G}$ is the symmetry group of the austenitic phase. For $\mathrm{Ni}_{2} \mathrm{MnGa}$, the symmetry group $\mathrm{G}$ is the cubic symmetry group, the group of proper rotations leaving the cube invariant. Since the energy density is invariant with respect to the direction of the magnetization [3], we also have that

$$
\varphi(F, m, \theta)=\varphi(F,-m, \theta) .
$$

At a fixed temperature below the martensitic transformation temperature, the energy density $\varphi$ is minimized at

$$
\operatorname{SO}(3)\left[U_{1}(\theta), \pm m_{1}(\theta)\right] \cup \ldots \cup \operatorname{SO}(3)\left[U_{n}(\theta), \pm m_{n}(\theta)\right]
$$

where the $U_{i}$ 's are the symmetry-related transformation matrices for the martensite and the $m_{i}$ 's are the corresponding preferred directions of magnetization (the easy axes).

Crystalline $\mathrm{Ni}_{2} \mathrm{MnGa}$ transforms from a high temperature phase with cubic symmetry to a low temperature phase with tetragonal symmetry [1,2]. If the reference state is taken to be the $\mathrm{Ni}_{2} \mathrm{MnGa}$ crystal in its cubic phase at the martensitic transformation temperature, then the martensitic transformation matrices with their associated easy axes of magnetization can be given by $[1,3]$

$$
\begin{gathered}
U_{1}=\left(\begin{array}{lll}
\beta & 0 & 0 \\
0 & \alpha & 0 \\
0 & 0 & \alpha
\end{array}\right), m_{1}=m_{s}\left(\begin{array}{l}
1 \\
0 \\
0
\end{array}\right), \quad U_{2}=\left(\begin{array}{ccc}
\alpha & 0 & 0 \\
0 & \beta & 0 \\
0 & 0 & \alpha
\end{array}\right), \quad m_{2}=m_{s}\left(\begin{array}{l}
0 \\
1 \\
0
\end{array}\right), \\
U_{3}=\left(\begin{array}{lll}
\alpha & 0 & 0 \\
0 & \alpha & 0 \\
0 & 0 & \beta
\end{array}\right), \quad m_{3}=m_{s}\left(\begin{array}{l}
0 \\
0 \\
1
\end{array}\right),
\end{gathered}
$$

where $\alpha=1.0163$ and $\beta=0.9555$ at the martensitic transformation temperature and where $m_{s}$ is the saturation magnetization.

We model the sum of the elastic and anisotropic energy density $\varphi(F, m, \theta)$ for $\mathrm{Ni}_{2} \mathrm{MnGa}$ by

$$
\varphi(F, m, \theta)=\varphi_{1}(F, \theta)+\varphi_{2}(F, m, \theta),
$$

where $\varphi_{1}(F, \theta)$ is an elastic energy density for a martensitic crystal that undergoes a cubic to tetragonal transformation (see [7]), and

$$
\varphi_{2}(F, m, \theta)=\frac{\kappa_{u}}{\alpha^{2}-\beta^{2}}\left|\frac{m \cdot B m}{m \cdot m}-\beta^{2}\right|,
$$

where $B=F F^{T}$ is the left Cauchy-Green strain and $\kappa_{u}$ is the magnetic anisotropic constant $[1,3]$. We note that for $\theta$ below the martensitic transformation temperature, $\varphi_{1}(F, \theta)$ is constructed to be minimized by the deformation gradients

$$
\operatorname{SO}(3) U_{1}(\theta) \cup \operatorname{SO}(3) U_{2}(\theta) \cup \operatorname{SO}(3) U_{3}(\theta)
$$

and that $\varphi_{2}\left(U_{i}, m, \theta\right)$ is minimized at $m= \pm m_{s} e_{i}$. Thus, the energy density $\varphi(F, m, \theta)$ is minimized on (2.7). 


\section{A THIN FILM MODEL FOR FERROMAGNETIC SHAPE MEMORY CRYSTALS}

Our thin film model for ferromagnetic shape memory crystals combines the rigorously derived thin film model for martensitic crystals of $[4,10]$ with the rigorously derived thin film model for ferromagnetic crystals of [11]. Our model is that local minima (stable equilibria) $(u, m)$ of the bulk energy $e^{(\delta)}(u, m)$ are deformations satisfying

$$
u\left(x_{1}, x_{2}, x_{3}\right)=y\left(x_{1}, x_{2}\right)+b\left(x_{1}, x_{2}\right) x_{3}+o(1) \text { for }\left(x_{1}, x_{2}\right) \in S \text { and } x_{3} \in(-\delta / 2, \delta / 2)
$$

and magnetizations satisfying

$$
m\left(u\left(x_{1}, x_{2}, x_{3}\right)\right)=M\left(x_{1}, x_{2}\right)+o(1) \text { for }\left(x_{1}, x_{2}\right) \in S \text { and } x_{3} \in(-\delta / 2, \delta / 2)
$$

where the deformations $y\left(x_{1}, x_{2}\right): S \rightarrow \mathrm{R}^{3}$ and $b\left(x_{1}, x_{2}\right): S \rightarrow \mathrm{R}^{3}$ and the magnetizations $M\left(x_{1}, x_{2}\right): S \rightarrow \mathrm{R}^{3}$ are local minima (stable equilibria) $(y, b, M)$ of the energy density per unit thickness

$$
\begin{aligned}
E(y, b, M) & =\int_{S} \kappa\left(\left|D^{2} y\left(x_{1}, x_{2}\right)\right|^{2}+2\left|\nabla b\left(x_{1}, x_{2}\right)\right|^{2}\right) d x_{1} d x_{2} \\
& +\int_{S} \varphi\left(\left[y_{, 1}\left|y_{, 2}\right| b\right], M, \theta\right) d x_{1} d x_{2} \\
& +\int_{S}\left\{\mu\left|\nabla_{y(S)} M\right|^{2}-M\left(x_{1}, x_{2}\right) \cdot h\left[y\left(x_{1}, x_{2}\right)\right]\right\} \operatorname{det}\left[y_{, 1}\left|y_{, 2}\right| b\right] d x_{1} d x_{2} \\
& +\frac{1}{2} \int_{S} M_{n}^{2} \operatorname{det}\left[y_{, 1}\left|y_{, 2}\right| b\right] d x_{1} d x_{2} .
\end{aligned}
$$

We denoted above by $\left[y_{, 1}\left|y_{, 2}\right| b\right]$ the deformation gradient matrix constructed from the column vectors $y_{, 1}, y_{, 2}$, and $b$. We also denoted by $\nabla_{y(S)} M$ the projection of the gradient $\nabla M$ onto the tangent plane of the surface $y(S)$. We note that the magnetization energy $e_{\text {mag }}^{(\delta)}(u, m)$ has been modeled as in [11] by $\frac{1}{2} \int_{S} M_{n}^{2} \operatorname{det}\left[y_{, 1}\left|y_{, 2}\right| b\right] d x_{1} d x_{2}$ where the component of the magnetization normal to the film surface is given by $M_{n}\left(x_{1}, x_{2}\right)=M\left(x_{1}, x_{2}\right) \cdot n\left[y\left(x_{1}, x_{2}\right)\right]$ with $n\left[y\left(x_{1}, x_{2}\right)\right]$ being the normal to the film surface $y(S)$ at $y\left(x_{1}, x_{2}\right)$.

We will present computational results for a film attached to the substrate at two opposite edges and free on the other two edges by assuming that

$$
\Omega_{\delta}=\left\{\left(x_{1}, x_{2}, x_{3}\right): 0<x_{1}<1,0<x_{2}<1,-\delta / 2<x_{3}<\delta / 2\right\} .
$$

We further assume that the applied magnetic field is in the $z_{1}-z_{3}$ plane

$$
h(z)=\left(h_{1}\left(z_{1}, z_{3}\right), 0, h_{3}\left(z_{1}, z_{3}\right)\right) \text { for } z \in \mathrm{R}^{3},
$$

and we seek energy-minimizing deformations and magnetizations of the form

$$
u(x)=\left(u_{1}\left(x_{1}, x_{3}\right), \alpha x_{2}, u_{3}\left(x_{1}, x_{3}\right)\right) \text { and } m(u(x))=\left(m_{1}(u(x)), 0, m_{3}(u(x))\right) .
$$


Under this constraint, our model is that local minima (stable equilibria) $(u, m)$ of the bulk energy $e^{(\delta)}(u, m)$ are deformations of the form

for

$$
u\left(x_{1}, x_{2}, x_{3}\right)=y\left(x_{1}, x_{2}\right)+b\left(x_{1}, x_{2}\right) x_{3}+o(1) \text { for } 0<x_{1}<1,0<x_{2}<1,-\delta / 2<x_{3}<\delta / 2,
$$

(3.2) $y\left(x_{1}, x_{2}\right)=\left(y_{1}\left(x_{1}\right), \alpha x_{2}, y_{3}\left(x_{1}\right)\right)$ and $b\left(x_{1}, x_{2}\right)=\left(b_{1}\left(x_{1}\right), 0, b_{3}\left(x_{1}\right)\right), 0<x_{1}<1,0<x_{2}<1$, and magnetizations of the form

$$
m\left(u\left(x_{1}, x_{2}, x_{3}\right)\right)=M\left(x_{1}, x_{2}\right)+o(1) \text { for } 0<x_{1}<1,0<x_{2}<1,-\delta / 2<x_{3}<\delta / 2,
$$

for

$$
M\left(x_{1}, x_{2}\right)=\left(M_{1}\left(x_{1}\right), 0, M_{3}\left(x_{1}\right)\right) \text { for } 0<x_{1}<1,0<x_{2}<1,
$$

where $(y, b, M)$ are local minima (stable equilibria) of the energy density per unit thickness given by (3.1).

We can formulate this problem as a one-dimensional problem by introducing

$$
\tilde{y}\left(x_{1}\right)=\left(y_{1}\left(x_{1}\right), 0, y_{3}\left(x_{1}\right)\right), \quad \tilde{b}\left(x_{1}\right)=\left(b_{1}\left(x_{1}\right), 0, b_{3}\left(x_{1}\right)\right), \quad \tilde{M}\left(x_{1}\right)=\left(M_{1}\left(x_{1}\right), 0, M_{3}\left(x_{1}\right)\right),
$$

and the free energy

$$
\begin{aligned}
& \tilde{E}(\tilde{y}, \tilde{b}, \tilde{M})=\int_{0}^{1} \kappa\left\{\left|\tilde{y}^{\prime \prime}\right|^{2}+2\left|\tilde{b}^{\prime}\right|^{2}\right\}+\varphi\left(\left[\tilde{y}_{, 1}\left|\alpha e_{2}\right| \tilde{b}\right], \tilde{M}, \theta\right) d x_{1} \\
& \quad+\int_{0}^{1}\left\{\mu\left|\nabla_{y(S)} M\right|^{2}-\tilde{M}\left(x_{1}\right) \cdot h\left(\tilde{y}\left(x_{1}\right)\right)\right\} \operatorname{det}\left[\tilde{y}_{, 1}\left|\alpha e_{2}\right| \tilde{b}\right] d x_{1} \\
& \quad+\frac{1}{2} \int_{0}^{1} \frac{\left(\mathrm{y}_{1}^{\prime} M_{3}-y_{3}^{\prime} M_{1}\right)^{2}}{\left(\mathrm{y}_{1}^{\prime}\right)^{2}+\left(y_{3}^{\prime}\right)^{2}} \operatorname{det}\left[\tilde{y}_{, 1}\left|\alpha e_{2}\right| \tilde{b}\right] d x_{1} .
\end{aligned}
$$

We can see that $\tilde{E}(\tilde{y}, \tilde{b}, \tilde{M})=E(y, b, M)$ by checking that all of the terms in the integrand of (3.1) depend only on $x_{1}$ for deformations and magnetizations of the form (3.2) and (3.3). We note that

$$
\left[\tilde{y}_{, 1}\left|\tilde{y}_{, 2}\right| b\right]=\left[y^{\prime}\left|\alpha e_{2}\right| b\right]=\left[\begin{array}{ccc}
y_{1}^{\prime} & 0 & b_{1} \\
0 & \alpha & 0 \\
y_{3}^{\prime} & 0 & b_{3}
\end{array}\right] .
$$

We recall that the magnetization $m$ is constrained to satisfy the saturation condition (2.2), so

$$
\left|\tilde{M}\left(x_{1}\right)\right|=m_{s} \text { for } 0<x_{1}<1 \text {. }
$$

For our computations with the thin film energy (3.5), we use the frame-indifferent free energy density

$$
\varphi(F, m, \theta)=\varphi_{1}(F, \theta)+\varphi_{2}(F, m, \theta),
$$

with the elastic energy density below the transformation temperature being given for deformation gradients (3.6) by

$$
\varphi_{1}(F, \theta)=c_{1}\left(\mathrm{~F}_{11} \mathrm{~F}_{33}-\mathrm{F}_{13} \mathrm{~F}_{31}-\alpha \beta\right)^{2}+c_{2}\left(C_{11}+C_{33}-\left(\alpha^{2}+\beta^{2}\right)\right)^{2}+c_{3} C_{13}^{2}+c_{4}\left(C_{11} C_{33}-\alpha^{2} \beta^{2}\right)^{2},
$$

where $c_{2}, c_{3}$, and $c_{4}$ are positive elastic moduli, $c_{1}$ is a positive constant chosen to ensure that the deformation is orientation preserving [9], and $C=F^{T} F$ is the right Cauchy-Green strain. The anisotropic free energy density below the transformation temperature is given as above by 


$$
\varphi_{2}(F, m, \theta)=\frac{\kappa_{u}}{\alpha^{2}-\beta^{2}}\left|\frac{m \cdot B m}{m \cdot m}-\beta^{2}\right| .
$$

We present the results of our computational investigation into the control of the deformation of the thin film by the application of a magnetic field. We start our computation with the film in the variant $U_{1}$ with magnetization $m=m_{1}$ and attached to the substrate at the edges $x_{1}=0$ and $x_{1}=1$. More precisely, the initial deformation satisfies

$$
y\left(x_{1}, x_{2}\right)=\left(\beta x_{1}, \alpha x_{2}, 0\right) \text { and } b\left(x_{1}, x_{2}\right)=(0,0, \alpha) \text { for } 0<x_{1}<1 \text { and } 0<x_{2}<1,
$$

and the initial magnetization satisfies

$$
M\left(x_{1}, x_{2}\right)=m_{s}(1,0,0) \text { for } 0<x_{1}<1 \text { and } 0<x_{2}<1 .
$$

The film is attached to the substrate at the edges $x_{1}=0$ and $x_{1}=1$, so the deformation $y\left(x_{1}, x_{2}\right)$ is constrained to satisfy the boundary condition

$$
y\left(0, x_{2}\right)=\left(0, \alpha x_{2}, 0\right) \text { and } y\left(1, x_{2}\right)=\left(\beta, \alpha x_{2}, 0\right) \text { for } 0<x_{2}<1 .
$$

We note that the initial deformation (3.8) and magnetization (3.9) minimize the free energy (3.1) among all deformations and magnetizations satisfying the boundary condition (3.10) and the saturation condition (3.7). We present computational results for the minimization of the free energy (3.1) as the applied magnetic field $H$ for

$$
h(z)=(0,0, H) \text { for } z \in \mathrm{R}^{3}
$$

is varied. Our computational results simulate the field induced tunnel proposed in [12].
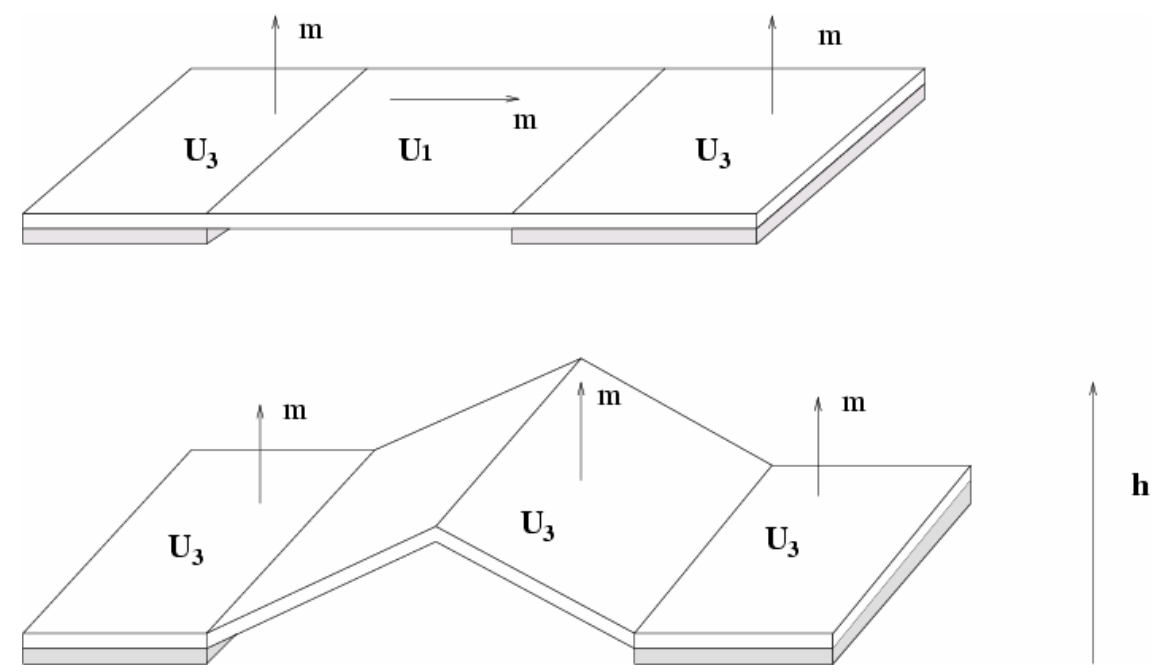

Figure 2. Field induced tunnel.

\section{NUMERICAL APPROXIMATION}

We start the numerical approximation with the applied magnetic field of the form (3.11) for field strength $H=0$ and the deformation (3.8) and magnetization (3.9). We then increase the field strength $H$ incrementally and at each new value of $H$ we minimize the free energy (3.5) by a nonlinear version of the Polak-Ribiere conjugate gradient method [13] with the initial iterate $(\tilde{y}, \tilde{b}, \tilde{M})$ being the local minima obtained at the previous value of $H$. After setting 
$\mu=0$ and the applied magnetic field of the form (3.11), the energy density $\tilde{E}(\tilde{y}, \tilde{b}, \tilde{M})$ takes the form

$$
\begin{aligned}
\tilde{E}(\tilde{y}, \tilde{b}, \tilde{M}) & =\int_{0}^{1} \kappa\left\{\left|\tilde{y}^{\prime \prime}\right|^{2}+2\left|\tilde{b}^{\prime}\right|^{2}\right\}+\varphi\left(\left[\tilde{y}_{, 1}\left|\alpha e_{2}\right| \tilde{b}\right], \tilde{M}, \theta\right) d x_{1} \\
& -\int_{0}^{1} M_{3}\left(x_{1}\right) H \operatorname{det}\left[\tilde{y}_{, 1}\left|\alpha e_{2}\right| \tilde{b}\right] d x_{1} \\
& +\frac{1}{2} \int_{0}^{1} \frac{\left(\mathrm{y}_{1}^{\prime} M_{3}-y_{3}^{\prime} M_{1}\right)^{2}}{\left(\mathrm{y}_{1}^{\prime}\right)^{2}+\left(y_{3}^{\prime}\right)^{2}} \operatorname{det}\left[\tilde{y}_{, 1}\left|\alpha e_{2}\right| \tilde{b}\right] d x_{1} .
\end{aligned}
$$

We approximate $\tilde{y}\left(x_{1}\right)=\left(y_{1}\left(x_{1}\right), 0, y_{3}\left(x_{1}\right)\right)$ by continuous, piecewise linear finite element functions on a uniform mesh that satisfy the boundary conditions

$$
y_{1}(0)=0, \quad y_{1}(1)=\beta, \quad y_{3}(0)=0, \quad y_{3}(0)=0 .
$$

We approximate $\tilde{b}\left(x_{1}\right)=\left(b_{1}\left(x_{1}\right), 0, b_{3}\left(x_{1}\right)\right)$ by piecewise constant finite element functions on the same uniform mesh of dimension $1 / N$, and we approximate $\tilde{M}\left(x_{1}\right)=\left(M_{1}\left(x_{1}\right), 0, M_{3}\left(x_{1}\right)\right)$ by piecewise constant finite element functions on the same uniform mesh that also satisfy the saturation condition (3.7).

The "strain gradient" surface energy $\int_{0}^{1} \kappa\left\{\left|\tilde{y}^{\prime \prime}\right|^{2}+2\left|\tilde{b}^{\prime}\right|^{2}\right\} d x_{1}$ is not finite for general continuous, piecewise linear $\tilde{y}\left(x_{1}\right)$ and piecewise constant $\tilde{b}\left(x_{1}\right)$. In our computations, we use the "total variation" surface energy (2.3) which gives for this model

$$
\sum_{e}\left(\left|\llbracket \tilde{y}^{\prime} \rrbracket_{e}\right|^{2}+2\left|\llbracket \tilde{b}^{\prime} \rrbracket_{e}\right|^{2}\right)^{1 / 2}
$$

where $e$ denotes the nodes of the mesh and where $\llbracket \tilde{y}^{\prime} \rrbracket_{e}$ and $\llbracket \tilde{b} \rrbracket_{e}$ denote the jump of $\tilde{y}$ and $\tilde{b}$ across the mesh node $e$.

\section{RESULTS AND DISCUSSION}

As an initial test that our model can predict qualitative behavior of a ferromagnetic shape memory alloy, we used in our computations non-physical dimensionless coefficients. We set the elastic moduli $c_{1}=c_{2}=c_{3}=c_{4}=30$, the magnetic anisotropic constant $\kappa_{u}=40$, the surface energy coefficient $\kappa=2 \times 10^{-2}$, and the saturation magnetization $m_{s}=1$. We use the transformation matrices given earlier for $\mathrm{Ni}_{2} \mathrm{MnGa}$ with $\alpha=1.0163$ and $\beta=0.9555$. We present results in Figure 3 for the deflection of the film by increasing the dimensionless magnetic field from $H=0$ to $H=3$ with increments $\Delta H=0.01$. Results are presented for a uniform mesh of size $1 / 36$.

We can observe in Figure 3 that as the applied magnetic field is increased from $H=0$, the film remains stationary but the magnetization starts to tilt in the direction of the applied field so as to reduce the interaction energy. At a critical applied magnetic field, the film begins to deflect. 


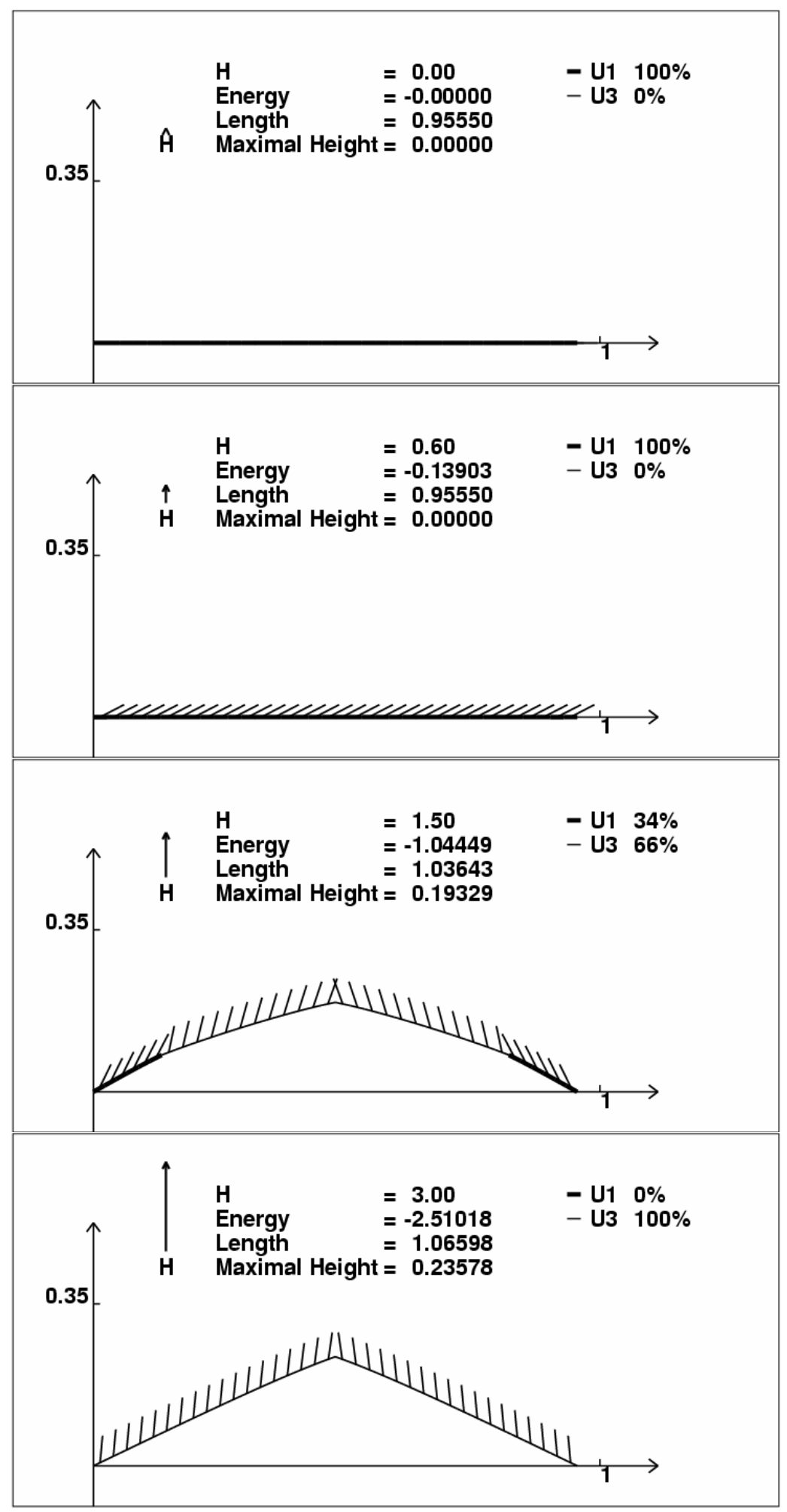

Figure 3. Deflection of the film by increasing the applied magnetic field. 
To ensure that the film deflect upwards rather than downward (the free energy is symmetric with respect to a reflection of the deformation about the initial deformation), we added the penalty term

$$
v \sum_{e}\left|y_{3}(e)\right|_{-}^{2}
$$

to the free energy, where $\left|y_{3}(e)\right|_{-}=0$ if $y_{3}(e)>0$ and $\left|y_{3}(e)\right|_{-}=y_{3}(e)$ if $y_{3}(e)<0$. This term is zero once the film has been deflected upward.

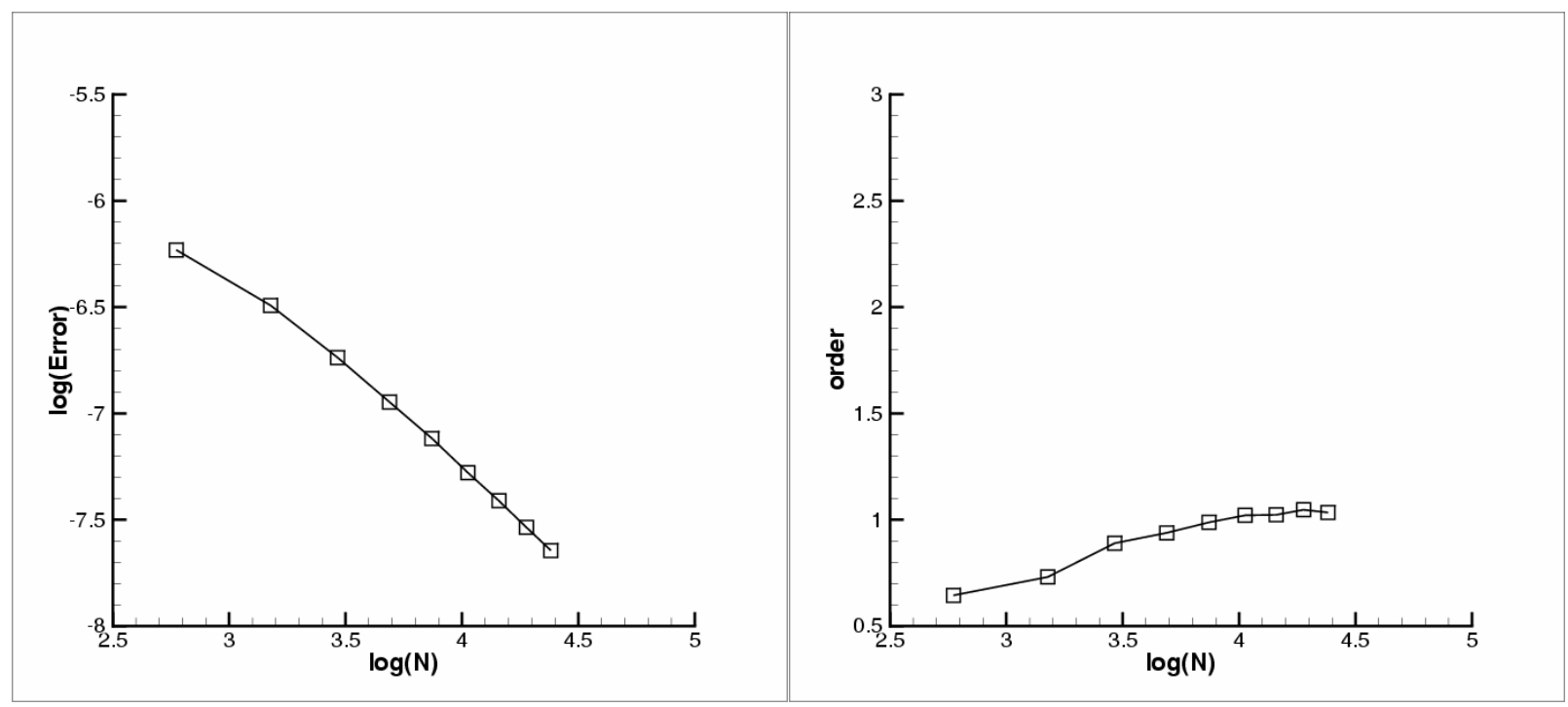

Figure 4. First order convergence of the error in the free energy as the mesh size $1 / N \rightarrow 0$.

\section{CONCLUSIONS}

We have proposed a model for the deformation of single crystal ferromagnetic shape memory thin film, and we have developed this model to the fit the transformation matrices of the $\mathrm{Ni}_{2} \mathrm{MnGa}$ alloy. Our finite element numerical approximation and computation demonstrates that the model predicts the deflection of a single crystal ferromagnetic shape memory thin film by the application of a magnetic field.

\section{ACKNOWLEDGEMENTS}

This work was supported in part by DMS-0304326, the Institute for Mathematics and Its Applications, and by the Minnesota Supercomputer Institute.

\section{REFERENCES}

[1] R.D. James and M. Wuttig, Philos. Mag. A 77, 1273 (1998). 
[2] J.W. Dong, J.Q. Xie, J. Lu, C. Adelmann, C.J. Palmstrøm, J. Cui, Q. Pan, T.W. Shield, R.D. James, and S. McKernan, J. Appl. Phys. 95, 2593 (2004).

[3] R.D. James and D. Kinderlehrer, Philos. Mag. A77, 1273 (1993).

[4] P. Belik and Luskin, Interfaces Free Bound. 4, 71 (2002).

[5] P. Belik and M. Luskin, Math. Models Methods Appl. Sci. 14, 1585 (2004).

[6] P. Belik and M. Luskin, Multiscale Model. Simul. 3, 764 (2004).

[7] M. Luskin, Acta Numer., 5, 191 (1996).

[8] R.D. James and K.F. Hane, Acta. Mater. 48, 197 (2000).

[9] M. Gurtin: Topics in Finite Elasticty. Philadelphia: SIAM; 1981.

[10] K. Bhattacharya and R. D. James, J. Mech. Phys. Solids 47, 531 (1999).

[11] G. Gioia and R. D. James, Proc. R. Soc. Lond. 453, 213 (1997).

[12] K. Bhattacharya, A. DeSimone, K.F. Hane, R.D. James, and C.J. Palmstrøm, Mater. Sci. Eng. A273-275, 685 (1999).

[13] P. Belik, T. Brule, and M. Luskin, Math. Model. Numer. Anal. 35, 525 (2001). 